\title{
V-shaped electro-optic response observed in a chiral ferroelectric smectic liquid crystal
}

\author{
V. Manjuladevi, ${ }^{1,2}$ Yu. P. Panarin, ${ }^{1,3}$ Jang-Kun Song, ${ }^{1}$ J. K. Vij, ${ }^{1, a)}$ and B. K. Sadashiva ${ }^{4}$ \\ ${ }^{1}$ Department of Electronic and Electrical Engineering, Trinity College Dublin, University of Dublin, \\ Dublin 2, Ireland \\ ${ }^{2}$ Department of Physics, Birla Institute of Technology and Science, Pilani, Rajasthan 333031, India \\ ${ }^{3}$ Department of Electronic and Communication Engineering, Dublin Institute of Technology, Dublin 8, Ireland \\ ${ }^{4}$ Liquid Crystal Laboratory, Raman Research Institute, C.V. Raman Avenue, Bangalore 560080, India
}

(Received 26 June 2008; accepted 22 July 2008; published online 3 September 2008)

\begin{abstract}
We report on the observation of $\mathrm{V}$-shaped switching in a ferroelectric liquid crystal cell over a wide range of temperatures. Results of the optical transmittance in the visible region give us the helical pitch for various temperatures of the ferroelectric liquid crystalline compound used. We show that the helical pitch, in addition to the spontaneous polarization $\left(P_{S}\right)$ and thickness of the alignment layer of the cell, is an important factor in giving $\mathrm{V}$-shaped switching. A longer or helical compensated mixture gives a better V-shaped switching. (C) 2008 American Institute of Physics.

[DOI: $10.1063 / 1.2970039$ ]
\end{abstract}

The origin of V-shaped (thresholdless) switching observed in ferroelectric smectic $\mathrm{C}^{*}\left(\mathrm{SmC}^{*}\right)$ liquid crystals has been a subject of debate for almost a decade. ${ }^{1-8} \mathrm{~V}$-shaped switching in surface stabilized ferroelectric liquid crystals (FLCs) is an attractive electro-optic effect for display applications. ${ }^{9-11}$ Many groups have explained this electrooptic response using different models. ${ }^{1-8}$ Using a detailed experimental study on some FLC mixtures with compensated helix, we have shown ${ }^{12,13}$ that the electrostatic model ${ }^{3-5}$ explains the electro-optic switching characteristics of VFLCs (V-shaped FLCs) extremely well, and that not only the high spontaneous polarization $P_{S}$ but also the thickness of alignment layer play an important role in obtaining a good V-shaped switching. The electrostatic model stiffens the directors such that, on the average, the tilt plane containing the layer normal is normal to the plane of substrates and $P_{S}$ while in the smectic layer plane is directed along the substrate. This arises from an increase in the elastic constant and consequently leads to stiffness in the $c$ directors with an increase in $P_{S}$, as was initially suggested by Okano. ${ }^{14}$ This was recognized by Rudquist et $a l^{3}$ and later by Clark and co-workers ${ }^{4,5}$ for realizing V-shaped switching in FLC cells. Hasegawa et al. $^{11}$ also discussed the importance of large $P_{S}$ in obtaining V-shaped switching with a contrast ratio (CR) greater than 100. Blinov et al. ${ }^{15}$ considered V-shaped switching in terms of an electrical equivalent circuit of the cell and derived an equation for the hysteresis inversion frequency using a triangular wave as applied signal and they suggested methods of increasing this frequency. It may be remarked here that although a triangular wave is used in demonstrating this phenomenon, in the real displays, nevertheless, pulse switching is used in the addressing-schemes. The switching speeds of the liquid crystals consequently come into play and the inversion frequency so derived is inapplicable. In fact, Cassio used MGC Y102 mixture and obtained $100 \mu$ s response time at room temperature by applying a few volts in this cell geometry. ${ }^{16}$

\footnotetext{
a) Author to whom correspondence should be addressed. Electronic mail: jvij@tcd.ie.
}

In this letter we present an additional characteristic of the V-shaped switching by observing the electro-optic switching behavior of a pure compound exhibiting $\mathrm{SmC}^{*}$ phase over a wide range of temperatures; the length of helical pitch is shown to play an important role. The material used is $[2 S, 3 S] \quad 4^{\prime}$-(2-chloro3-methylpentyloxycarbonyl) phenyl trans-4"- $n$-decyloxycinnamate abbreviated as CMPD and its chemical formula is given in Fig. 1 and synthesis described..$^{17}$ The phase sequence is I $90{ }^{\circ} \mathrm{C} \mathrm{SmA} 70{ }^{\circ} \mathrm{C}$ SmC* $44^{\circ} \mathrm{C}$ Cr. Upon cooling, the liquid crystal supercools and remains in the $\mathrm{SmC}^{*}$ phase down to room temperature. The helical pitch was measured by using freestanding films of $\sim 60 \mu \mathrm{m}$ thickness mounted on a UV/visible near-IR spectrometer (PerkinElmer Lambda 900). Figure 2 shows the peak wavelength of the selective reflection as a function of temperature for the half-pitch band. As an example, the spectra corresponding to this band in the $\mathrm{SmC}^{*}$ phase at $54{ }^{\circ} \mathrm{C}$ are shown in the inset of Fig. 2; the full pitch band could not be measured as it remained out of the range of detectable wavelength of the spectrometer. The spontaneous polarization $\left(P_{S}\right)$ was measured using a method based on the measurement of repolarization current, described by Vaksman and Panarin. ${ }^{18}$ A $9 \mu \mathrm{m}$ planar cell was used for measuring the spontaneous polarization; the temperature dependence of $P_{S}$ in $\mathrm{SmC}^{*}$ is shown in Fig. 2. The electro-optic switching response observed at a frequency of $100 \mathrm{mHz}$ for various temperatures is shown in Fig. 3. The sample shows a good $\mathrm{V}$-shaped switching over a wide range of frequencies from $100 \mathrm{mHz}$ to $1 \mathrm{~Hz}$, although the response here is shown for a single frequency. The red horizontal lines at the bottom of the V-shaped curves correspond to the transmittance between

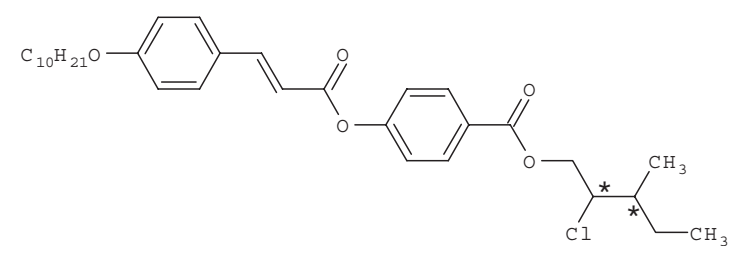

FIG. 1. The structural formula of the compound CMPD. 


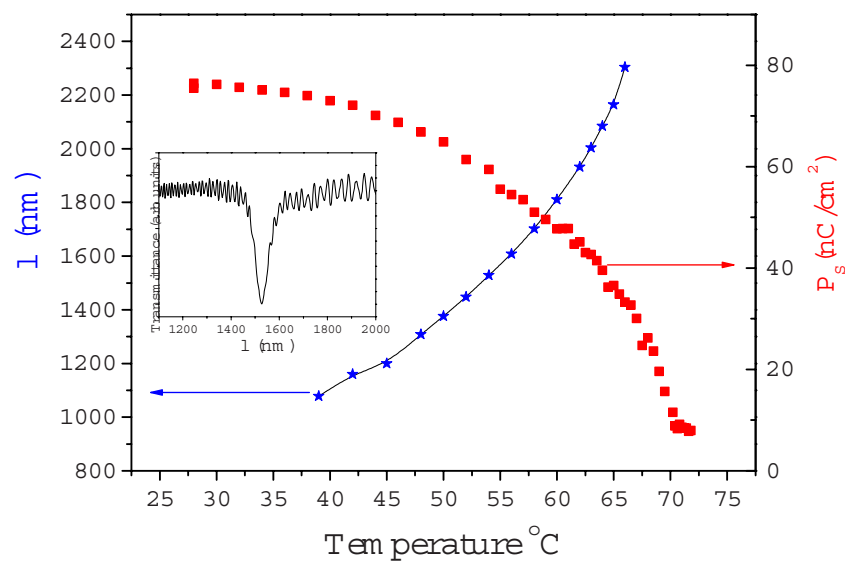

FIG. 2. (Color online) Temperature dependence of the half-pitch band of the reflection band of the compound CMPD in its $\mathrm{SmC}^{*}($ pitch $=\lambda / n$ ) phase and the spontaneous polarization $\left(P_{S}\right)$. The inset shows an example of the halfpitch peak observed in the transmission UV/visible spectroscopy. $n$ is the average refractive index assumed to be 1.5 .

the crossed polarizers when the applied electric field is switched off. The field off state is quite dark at higher temperatures, as seen in Fig. 3. The field off state shifts toward the brighter levels as the sample is cooled down toward the room temperature.

At higher frequencies, the electro-optic response shows positive hysteresis due to a delay in the switching. At low frequencies, the electro-optic response shows negative hysteresis due to transport of ions in the cell. The effect of ions on the appearance of low frequency hysteresis has been explained using a simple mathematical model by Panarin et al. ${ }^{1,19}$ Our previous measurements on FLC mixtures have established that the V-shaped switching gets distorted upon lowering either the spontaneous polarization or decreasing the alignment layer thickness. ${ }^{12,13}$ This can be explained using the "electrostatic" model. ${ }^{3,5} \mathrm{O}^{\prime}$ Callaghan ${ }^{6}$ has discussed the importance of this model in detail and has found the electrostatic energy to be $W_{0}=P_{S}^{2} d_{a} / \varepsilon_{a}\left[1+\left(\varepsilon / \varepsilon_{a}\right)\left(2 d_{a} / d\right)\right]$, which approximates to $P_{S}^{2} d_{a} / \varepsilon_{a}$.

Here $d_{a}$ and $d$ are the thicknesses of the alignment layer and the FLC layer, respectively. $\varepsilon$ and $\varepsilon_{a}$ are the permittivities of the FLC layer and the alignment layer, respectively. $W_{0}$ proportional to $P_{S}^{2}$ varies with temperature depending on $P_{S}$, as shown in Fig. 2. Hence, the CR of the cell at various temperatures plotted as a function of $W_{0}$ is shown in Fig. 4 as it varies with $W_{0}$. We define the two types of contrast ratios: (i) the dynamic contrast ratio obtained during the switching of cells, the same as the CR used in our previous paper, ${ }^{12}$ and (ii) the static CR that indicates the ratio of the brightness at large fields to the brightness at zero field in the static case. As explained above, the field off state shifts toward the brighter levels as the sample is cooled down toward the room temperature. Hence, the static CR differs from the dynamic $\mathrm{CR}$ especially at low temperatures. The dynamic CR decreases with a decrease in $W_{0}$. This is in agreement with our previous work. $^{12,13}$ The dynamic $\mathrm{CR}$ even at a temperature of approximately $44{ }^{\circ} \mathrm{C}$ is $\sim 18$, where the value of $P_{S}$ is $\sim 73 \mathrm{nC} / \mathrm{cm}^{2}$, again in agreement with our previous results on the binary mixtures of a chiral dopant and achiral smectic systems. ${ }^{12,13}$ However, the static CR shows a different behavior. At low values of $W_{0}$, it increases with $W_{0}$ but decreases for large $W_{0}$. The latter is not expected from the electrostatic model but is explained here in terms of the helical pitch. The helical pitch $p_{0}$ at $44{ }^{\circ} \mathrm{C}$ is $\sim 0.820 \mu \mathrm{m}$ but is not short enough to suddenly form a helical structure in a planar cell with thickness of $1.3 \mu \mathrm{m}$. Nevertheless, when the electric field is turned off the helical restoring force may affect the uniform director distribution, trying to form a sort
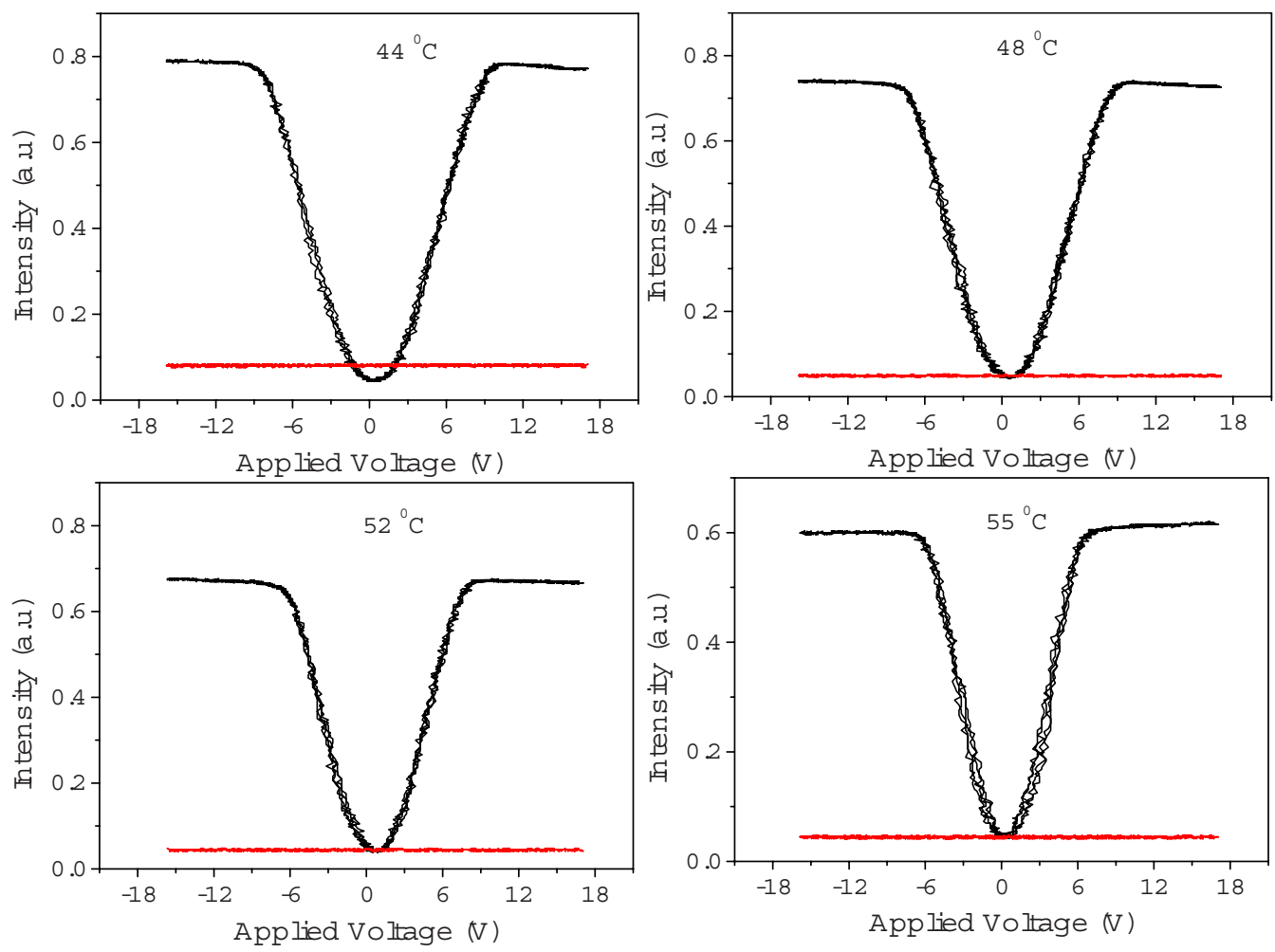

FIG. 3. (Color online) Electro-optic response of CMPD in a $1.3 \mu \mathrm{m}$ cell at various temperatures at frequency of $100 \mathrm{mHz}$. The red horizontal lines indicate the dark (field off) state. 


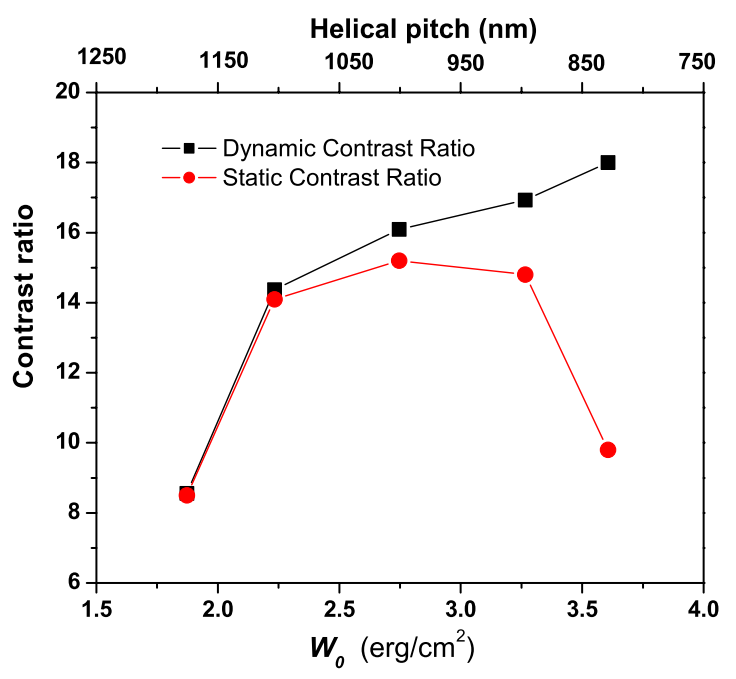

FIG. 4. (Color online) The two contrast ratios as a function of $W_{0}$ and of the helical pitch $p_{0}$ in a $1.3 \mu \mathrm{m}$ planar cell of CMPD.

of helical cluster/domain structure which appears as lines parallel to the smectic layers. Thus shorter helical pitch deteriorates the uniformity of the dark state which decreases the static CR; the latter is important if the dark state is to be maintained over a relatively longer period of time. It takes time of the order of several seconds or more to form such a distorted helical structure; hence, it does not appear when the field is varied and therefore it does not affect the dynamic $\mathrm{CR}$. This explains as to why the static contrast ratio is less than its corresponding dynamic one especially for lower pitch values, i.e., at lower temperatures. At higher temperatures, the pitch is longer, as shown in Fig. 2. The restoring force to form a helical structure is relatively lower as the pitch is longer and hence the dark state is relatively darker. The static CR in Fig. 4 is shown to decrease with decrease in the helical pitch. However, at higher pitch values, it goes down mainly due to $W_{0}$ which is dependent on the decrease in $P_{S}$ with increase in temperature. Ideally, the CR should be plotted for a constant $P_{S}$, which is somewhat valid for lower temperatures only (see Fig. 2).

We show the texture of the sample in the field off state at three temperatures in Fig. 5. At a temperature of $60^{\circ} \mathrm{C}, \mathrm{CR}$ drops down as expected because $P_{S}$ reduces to $48 \mathrm{nC} / \mathrm{cm}^{2}$. Even though at $44{ }^{\circ} \mathrm{C}$ the sample shows a good V-shaped switching, the field off state is rather brighter compared to the one at $52{ }^{\circ} \mathrm{C}$ (Fig. 3). This is due to the fact that the helical pitch of the CMPD decreases with decreasing temperature, leading to the domainlike structures due to the observation of pitch lines shown in Fig. 5(a). From the results of the experiments we find that the important factors to
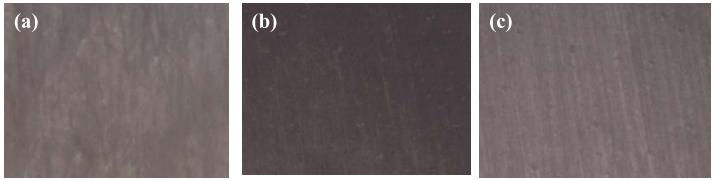

FIG. 5. (Color online) Textures of the $1.3 \mu \mathrm{m}$ thick planar cell containing the compound between the crossed polarizers in the field off state at temperatures of (a) $44^{\circ} \mathrm{C}$, (b) $52{ }^{\circ} \mathrm{C}$, and (c) $60{ }^{\circ} \mathrm{C}$.

achieve a good shaped V-switching are high $P_{S}$, thick alignment layer as found before, ${ }^{12,13}$ and a longer helical pitch of the FLC material as has been found here.

We thank the Science Foundation of Ireland (SFI) under Grant No. (02/IN.1/I031) for funding the research work in Dublin. We also thank Atsuo Fukuda for useful discussions and I. Kobayashi of Nissan Chemical Industries Japan for the gift of the aligning agent (RN1175).

${ }^{1}$ Yu. P. Panarin, Mol. Mater. 2, 137 (1993).

${ }^{2}$ S. Inui, N. Iimura, T. Suzuki, H. Iwane, K. Miyachi, Y. Takanishi, and A. Fukuda, J. Mater. Chem. 6, 671 (1996).

${ }^{3}$ P. Rudquist, J P. F. Lagerwall, M. Buivydas, F. Gouda, S. T. Lagerwall, N. A. Clark, J. E. Maclennan, R. Shao, D. A. Coleman, S. Bardin, T. Bellini, D. R. Link, G. Natale, M. A. Glaser, D. M. Walba, M. D. Wand, and X. -H. Chan, J. Mater. Chem. 9, 1257 (1999).

${ }^{4}$ N. A. Clark, D. Coleman, and J. E. Maclennan, Liq. Cryst. 27, 985 (2000)

${ }^{5}$ M. Copic, J. E. Maclennan, and N. A. Clark, Phys. Rev. E 65, 021708 (2002)

${ }^{6}$ M. J. O'Callaghan, Phys. Rev. E 67, 011710 (2003).

${ }^{7}$ L. M. Blinov, S. P. Palto, F. V. Podogornov, H. Moritake, and W. Haase, Liq. Cryst. 31, 61 (2004).

${ }^{8}$ S. Tzeng, T. Y. Lin, R. H. Haung, J. Wu, and S. Wu, Phys. Rev. E 70, 011712 (2004).

${ }^{9}$ T. Saishu, K. Takatoh, R. Iida, H. Nagata, and Y. Mori, SID International Symposium Digest Technical Papers 1996 (unpublished), 703.

${ }^{10}$ T. Yoshida, T. Tanaka, J. Ogura, H. Wakai, and H. Aoki, SID Inst. Symp. Digest Tech. Papers 1997 (unpublished), 841.

${ }^{11}$ R. Hasegawa, H. Fujiwara, H. Nagata, Y. Hara, T. Saishu, R. Fukushima, M. Akiyama, H. Okumura, and K. Takatoh, J. Soc. Inf. Disp. 9, 107 (2001).

${ }^{12}$ V. Manjuladevi, Yu. P. Panarin, and J. K. Vij, Appl. Phys. Lett. 91, 052911 (2007).

${ }^{13}$ V. Manjuladevi, J. K. Vij, and Yu. P. Panarin, Mol. Cryst. Liq. Cryst. 477, 717 (2007).

${ }^{14}$ K. Okano, Jpn. J. Appl. Phys., Part 1 25, 1846 (1986).

${ }^{15}$ L. M. Blinov, S. P. Palto, E. P. Pozhidaev, Yu. P. Bobyley, V. M. Shoshin, A. L. Andreev, F. V. Podgornov, and W. Hassse, Phys. Rev. E 71, 051715 (2005).

${ }^{16}$ Y Yoshida, T. Tanaka, J. Ogura, H. Wakai, and H. Aoki, SID Int. Symp. Digest Tech. Papers 28, 841 (1997).

${ }^{17}$ B. Shivkumar, B. K. Sadashiva, S. Krishna Prasad, and V. N. Raja, Ferroelectrics 114, 273 (1991).

${ }^{18}$ V. M. Vaksman and Yu. P. Panarin, Mol. Mater. 1, 147 (1992).

${ }^{19}$ Yu. P. Panarin, V. Panov, O. E. Kalinovskaya, and J. K. Vij, Ferroelectrics 246, 35 (2000). 